\title{
Reduction of Cortico-spinal Excitability by Transcranial Magnetic Stimulation at Predictable Timing
}

\author{
Tomohiko TAKEI, Toshihiro HASHIMOTO, Nobuhiro HAGURA, \\ Michikazu MATSUMURA, and Eiichi NAITO \\ Graduate School of Human and Environmental Studies, Kyoto University, Sakyo-ku, \\ Kyoto 606-8501, Japan
}

\begin{abstract}
Electrophysiological studies have shown that cortico-spinal excitability increases during the motor preparation period in reaction time (RT) paradigms. However, there is a line of contradictory evidence with transcranial magnetic stimulation (TMS) showing that its excitability is reduced during the preparation period. In these studies, the subjects can predict the TMS timings. Thus we investigated how the predictability of TMS timing affects cortico-spinal excitability. A single-pulse TMS was delivered to the hand section of the left motor cortex while seven right-handed subjects relaxed their hands in a flexed position. We prepared three conditions: (i) in the semi-PREDICTABLE condition, two visual stimuli at $500 \mathrm{~ms}$ interval were pre-
\end{abstract}

sented and the TMS was delivered either 0, 125, 250,375 , or 500 ms after the first stimulus; (ii) in the PREDICTABLE condition, the TMS was provided only at $500 \mathrm{~ms}$ after the first stimulus; (iii) in the UNPREDICTABLE condition, no visual cue preceded the TMS. We recorded motor evoked potentials (MEPs) from the wrist flexor and extensor muscles. We found a significant reduction of MEP amplitude in the flexor muscles in both the PREDICTABLE and semi-PREDICTABLE conditions, but not in the UNPREDICTABLE condition. These results showed that the predictability of TMS per se, without the preparation of motor outputs, can reduce cortico-spinal excitability. [The Japanese Journal of Physiology 55: 93-99, 2005]

Key words: transcranial magnetic stimulation (TMS), human motor cortex, cortico-spinal excitability, motor evoked potential.

The neuronal excitability in the human cortico-spinal
tract has been investigated by transcranial magnetic
stimulation (TMS) [1] during the reaction time period
[2]. Especially when warning (S1) and imperative (S2)
stimuli are presented and subjects must prepare and re-
spond to the S2 stimulus, it may be expected that the
neuronal excitability in the motor cortex increases to-
ward the initiation of a response as M1 neurons start
increasing their activity during the preparation period
[3-5]. However, a line of contradictory evidence shows
that its excitability was reduced during the S1-S2 re-
sponse preparation period [6-8]. In these studies, a
TMS was delivered during the S1-S2 period, which
was a relatively short and constant period (500 or 1,000
ms). In these constant situations, subjects could some- how predict the TMS timing. So we tested our hypothesis that cortico-spinal excitability may be reduced when subjects can predict TMS timing. More important, if the predictability of TMS was a crucial factor to reduce the excitability, we may expect this reduction to be observed even in a situation where no responses (no preparations) are required.

A single-pulse TMS was delivered to the hand section of the left motor cortex at either predictable or unpredictable timing while seven right-handed subjects relaxed their hands in a flexed position. We prepared three conditions. (i) In the semi-PREDICTABLE condition, two visual stimuli (S1 and S2) with a constant delay $(500 \mathrm{~ms})$ of $\mathrm{S} 2$ were presented, and the TMS was delivered at timings of $0,125,250,375$, or $500 \mathrm{~ms}$ after

Received on Oct 4, 2004; accepted on Apr 3, 2005; released online on Apr 28, 2005; DOI: 10.2170/jjphysiol.R2075 Abbreviations: ECR, extensor carpi radialis; EMG, electromyogram; FCR, flexor carpi radialis; M1, primary motor cortex; MT, motor threshold; MEP, motor evoked potential; TMS, transcranial magnetic stimulation.

Correspondence should be addressed to: Eiichi Naito, Graduate School of Human and Environmental Studies, Kyoto University, Sakyo-ku, Kyoto 606-8501, Japan. Phone: +81-75-753-6887, Fax:+81-75-753-7907, E-mail: eiichi.naito@neuro.mbox.media.kyoto-u.ac.jp 
S1. (ii) In the PREDICTABLE condition, the TMS was provided only at the fixed timing of $500 \mathrm{~ms}$ after S1. (iii) In the UNPREDICTABLE condition, no visual cue preceded the TMS. We recorded motor evoked potentials (MEP) from the flexor carpi radialis (FCR) and the extensor carpi radialis (ECR) muscles to evaluate cortico-spinal excitability. In the semi-PREDICTABLE condition, we tested whether cortico-spinal excitability would be reduced during the S1-S2 period even when no motor responses were required, in which TMS timing was somehow predictable. In the PREDICTABLE condition, the subjects could completely predict the TMS timing, whereas in the UNPREDICTABLE condition they could not. Thus by comparing MEP amplitudes between conditions, we may evaluate the effect of prediction on cortico-spinal excitability.

\section{MATERIALS AND METHODS}

Subjects. Seven healthy volunteers (all male; mean age 25.3 years, range 22-29 years) participated in the three conditions. All subjects were right-handed according to the Edinburgh scale [9] and had normal or corrected-to-normal visual acuity. The subjects were given a written informed consent form for the study, according to the Ethical Committee of the Graduate School of Human and Environmental Studies of Kyoto University. Five subjects participated in the semi-PREDICTABLE condition and three in the PREDICTABLE and UNPREDICTABLE conditions. One subject participated in all conditions.

Experimental conditions. Experiments were carried out in a shielded and dimly illuminated room. The subjects sat on a comfortable chair, and their heads were immobilized by a head stabilizer in a comfortable posture. Their right forearms were placed on a horizontal platform. We prepared three conditions. In the semiPREDICTABLE condition, the right hand was passively flexed and immobilized at 60 degrees. This wrist angle was selected because it corresponded to the angles when the subjects totally relaxed their hands with forearms pronated. This angle was fixed throughout the condition in order to keep a wrist posture constant, which affects the amplitude of motor evoked potentials (MEP) [10]. In the PREDICTABLE and UNPREDICTABLE conditions, we measured the displacement of wrist movement evoked by TMS by attaching a goniometer to the ulnar side of the right-hand carpal joint. This was done because we could not know the precise size of the evoked movements from MEP amplitudes. The right hand was not fixed, but was totally relaxed in a flexed position. The mean wrist angle was almost equal to that in the semi-PREDICTABLE condition a. semi-PREDICTABLE

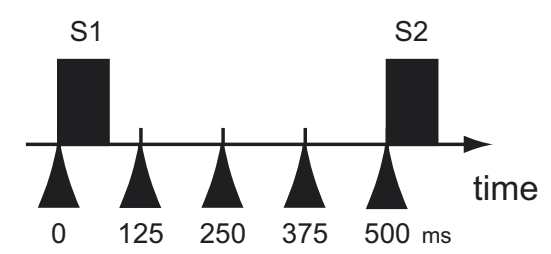

b. PREDICTABLE

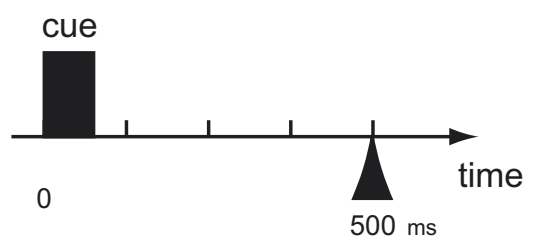

c. UNPREDICTABLE

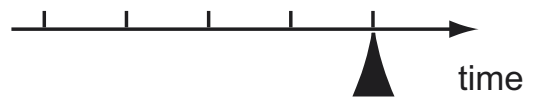

Fig. 1. Experimental conditions. In all experimental conditions, subjects with eyes open were required to completely relax and not prepare responses. a: In the semi-PREDICTABLE condition, an identical visual stimulus (light-emitting diode: LED; duration $100 \mathrm{~ms}$ ) was presented twice (S1 and S2) at an interval of 500 ms. A single-pulse TMS was delivered at $0,125,250,375$, or $500 \mathrm{~ms}$ after the onset of the $\mathrm{S} 1$ signal. b: In the PREDICTABLE condition, TMS was delivered at a fixed timing of $500 \mathrm{~ms}$ after the cue signal. No S2 was presented. c: In the UNPREDICTABLE condition, TMS was delivered at unexpectable timing without being preceded by a cue signal. The intertrial interval (ITI) was randomly selected from 10-20 s to prevent subjects from predicting the start of the next trial.

\section{(63.5 \pm 9.5 degrees).}

Trial conditions and experimental designs. In the semi-PREDICTABLE condition, a visual stimulus (a light-emitting diode: LED; a circle with a diameter of $5.0 \mathrm{~mm} ; 100 \mathrm{~ms}$ duration) $70 \mathrm{~cm}$ in front of the subjects at eye level was presented twice (S1 and S2) at an interval of $500 \mathrm{~ms}$ (Fig. 1a) in the same way as in the previous $\mathrm{S} 1-\mathrm{S} 2$ reaction time paradigms [6-8]. In the present study, however, the subjects were instructed to passively watch the S1 and S2 stimuli without preparing any motor responses. A single-pulse TMS was delivered at one of five latencies $(0,125,250,375$, and $500 \mathrm{~ms}$ ) after the onset of the S1 signal. In a control trial, the TMS was unexpectedly delivered after the $\mathrm{S} 2$ in the previous trial had been presented. One epoch then consisted of 6 types of trials $(0,125,250,375,500 \mathrm{~ms}$, and control). The TMS timing was randomized within an epoch and also counterbalanced within a block. The intertrial interval (ITI) was randomly selected from 10-20 s so that the subjects would not anticipate the 
timing of S1 after the previous trial was completed. Eventually, the subjects performed 36 trials (6 epochs) in one block and completed three blocks. No training was performed before the experiment.

In the PREDICTABLE condition, only one cue signal (LED) was presented. TMS was delivered at a fixed timing of $500 \mathrm{~ms}$ after the cue signal, which was similar to that of the semi-PREDICTABLE condition (Fig. 1b). The subjects had been informed of the timing of TMS in the PREDICTABLE condition (" $500 \mathrm{~ms}$ after the cue signal") before the experiment started. In the UNPREDICTABLE condition, TMS was delivered at an unexpectable timing without being preceded by a cue (LED) signal (Fig. 1c). The intertrial interval (ITI) was identical to that in the semi-PREDICTABLE condition. The subjects were required to make no voluntary responses in any trials. They performed 16 trials in a block and completed three blocks. Half the trials were assigned for the PREDICTABLE condition and the other half for the UNPREDICTABLE condition. The order of the PREDICTABLE or UNPREDICTABLE condition was counterbalanced within a block. In this situation, the subjects could not predict which condition was coming next.

Transcranial magnetic stimulation and stimulation procedure. A single-pulse magnetic stimulator (SMN-1200; maximum output intensity 0.67 Tesla; stimulus duration $90 \mu \mathrm{s}$; Nihon Kohden, Tokyo, Japan) with a double cone coil (YM133B; Nihon Kohden, Tokyo, Japan) was used in the experiments.

To precisely fix the TMS coil over the hand/wrist section of M1 throughout the experiments, we used the latest navigation system for TMS coils (Brainsight, Rogue Research, Montreal, Canada). We identified the most probable location of the left hand/wrist section of M1 in each subject. First we utilized functional magnetic resonance imaging (fMRI) data obtained from an independent group of 14 right-handed subjects to identify their M1 hand/wrist sections [11]. The coordinates of the sections corresponded to those in the standard brain space of the Montreal Neurological Institute. We then transformed these coordinates into the individual anatomical MRI space of each subject participating in the study. This scan contained five landmarks on an individual's head and face (nasion, an intratrageal notch of the left and right ears, a $6 \mathrm{~cm}$ dorsal point from nasion, and an $8 \mathrm{~cm}$ left point from nasion). These landmarks could provide their own relative positions in regard to an individual cortex. The individual MR image with five landmarks was registered with software (Brainsight flameless v.1.56, Rogue Research). During the experiments, reflective makers were attached to the TMS coil and to the subjects' heads. The software monitored their spa- tial locations with an infrared camera (Polaris hybrid position sensor, Northern Digital, Waterloo, Canada). Thus this system provides updated TMS coil position and orientation, informing an experimenter of the online monitored coil position and orientation relative to a subject's cortex. By adopting this technique, we could precisely identify the hand/wrist section of M1 where a TMS-evoked motor evoked potential (MEP) from the targeted wrist muscles in each subject. Once the position of the coil was determined, the coil was fixed by a goosenecked holder throughout the experiments.

To determine stimulus intensity and the orientation of the coil, we required the subjects to be totally relaxed with no muscular contraction and to fixate their eyes on the LED. The motor threshold (MT) was determined as the minimum intensity at which TMS evoked MEPs $(>50 \mu \mathrm{V})$ in at least half of 10 single TMSs [12] from both the flexor carpi radialis (FCR) and the extensor carpi radialis (ECR). The orientation of the coil was fixed to induce the current from posterior to anterior in the cortex. If this orientation was not optimal to evoke MEP, we rotated it 180 degrees. The TMS intensity in the experiments was finally set at $120 \%$ of an individual MT to successfully elicit the MEP in all trials.

Electoromyographic recording. Electromyograms (EMG) from the right FCR and the right ECR were recorded with paired surface $\mathrm{Ag} / \mathrm{AgCl}$ electrodes (Nihon Kohden, Tokyo, Japan). The hand muscles were selected because they have relatively lower MT [13]. Two electrodes were fixed about $2 \mathrm{~cm}$ apart on the skin over the bellies of the ECR and the FCR, which were identified by palpation during wrist flexion or extension movements. The impedance was reduced to below $10 \mathrm{k} \Omega$. The EMG activities were amplified 2,000 times (MEG6100-M, Nihon Kohden), band-pass filtered (5-1 kHz), sampled at $2,000 \mathrm{~Hz}$ by an A/D converter (ML795 Powerlab/16SP, ADInstruments Japan Inc., Nagoya, Japan) with 16-bit resolution, and stored in a personal computer for off-line analyses.

Data processing. MEP amplitude was analyzed off-line. MEP amplitude was defined as the difference between positive and negative peak amplitudes of the transient EMG response from 15 to $60 \mathrm{~ms}$ after the TMS. The displacement of evoked wrist movement, which was recorded by the goniometer (also sampled at $2,000 \mathrm{~Hz}$ ), was defined as the maximum angular change from the original wrist position. The onset of the movement was defined as the first deflection of the baseline.

Statistical processing. MEP amplitude data that exceeded \pm 3 standard deviation (SD) from a mean value in each subject were excluded from the analysis to eliminate the bias by outlier values. We pooled the data 
a

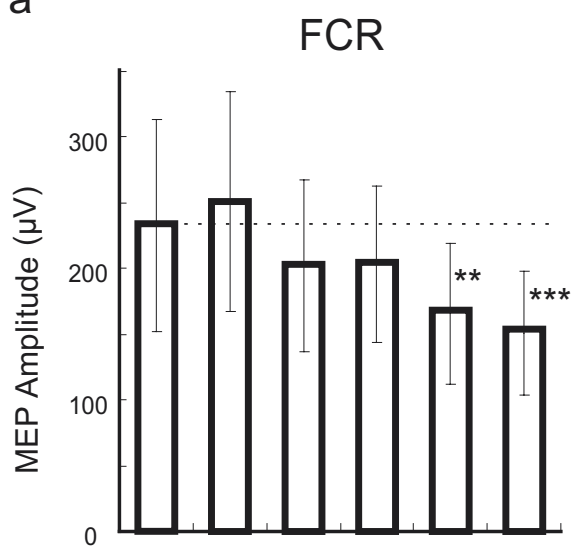

b

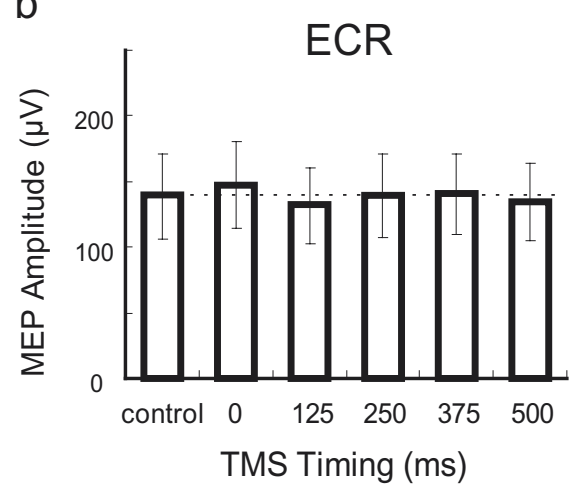

Fig. 2. Changes of MEP amplitudes in the semi-PREDICTABLE condition (a: FCR; b: ECR). MEP amplitudes were gradually reduced only in the FCR as TMS was delivered at a relatively later timing from the $S 1$ signal (a), whereas no reduction was observed in the ECR (b). Vertical lines indicate the \pm half value of standard deviation. Dashed lines indicate MEP amplitudes in the control condition.

from all subjects to increase the analysis sensitivity and found the same trend of results from each single subject as the one obtained from this analysis. We used the analysis of variance (ANOVA). This statistical analysis was carried out with distributed software (SPSS for Windows, ver. 10.0 J, SPSS Japan Inc., Tokyo, Japan).

\section{RESULTS}

\section{Semi-PREDICTABLE condition}

The MEPs were recorded both from the FCR and the ECR in all trials, and the subjects clearly reported that they had no intention of making any voluntary responses. The MEP amplitudes were gradually reduced as TMS was delivered at the relatively later timing from the $\mathrm{S} 1$ signal (Fig. 2). This reduction was observed only in the FCR, not in the ECR. These trends were consistently observed across subjects. A one-way ANOVA showed a significant reduction of MEP amplitudes only in the FCR $(F[5,524]=7.31, p<0.001)$. A Dunnett's test showed that the MEP amplitudes at 375 and $500 \mathrm{~ms}$ a
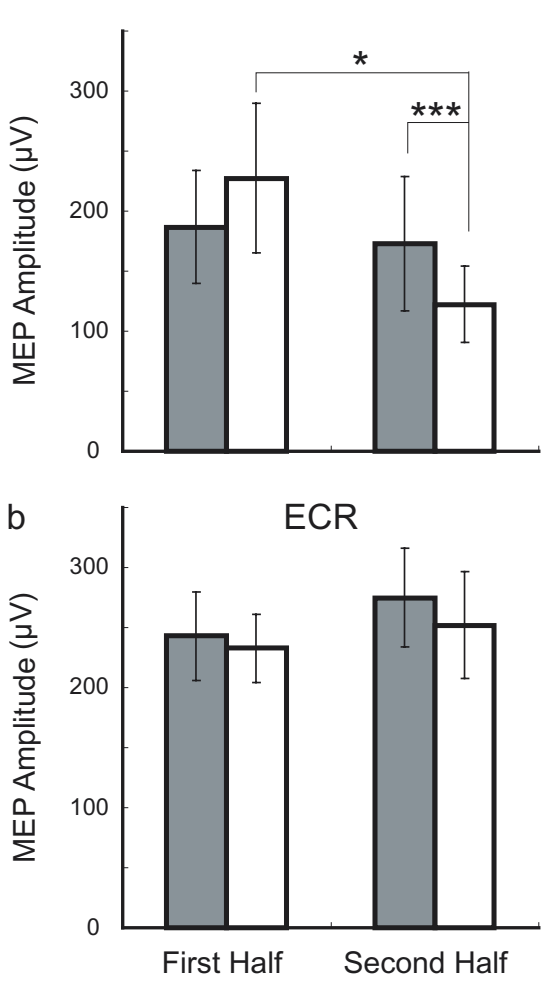

$\square$ UNPREDICTABLE $\square$ PREDICTABLE

Fig. 3. MEP amplitudes in the PREDICTABLE and UNPREDICTABLE conditions (a: FCR; b: ECR). a: In the first half of the trials, MEP amplitudes did not significantly differ between the PREDICTABLE and UNPREDICTABLE conditions ( $p=$ 0.57), whereas in the second half MEP amplitudes in the PREDICTABLE condition were significantly reduced when compared with those in the UNPREDICTABLE condition. $\mathbf{b}$ : No such difference was observed in the ECR.

were significantly reduced when compared with control trials ( $p<0.01$ and $p<0.001$, respectively). In contrast, MEP amplitudes in the ECR did not differ, regardless of TMS timing ( $p=0.66)$.

\section{PREDICTABLE and UNPREDICTABLE condi- tions}

MEP amplitude. During this experiment, we noticed that MEP amplitudes from the FCR and the ECR changed between the first half of the trials and the second half (Fig. 3). Thus to precisely describe the changes of MEP amplitudes, we treated the data separately in both halves of the trials in the PREDICTABLE and UNPREDICTABLE conditions.

In the FCR, MEP amplitudes in the first half did not differ between the PREDICTABLE and UNPREDICTABLE conditions, whereas in the second half, MEP amplitudes in the PREDICTABLE condition were reduced when compared with those in the UNPREDICTABLE condition (Fig. 3a). These trends were consistently observed across subjects. A two-way ANOVA 
a

(i) UNPREDICTABLE

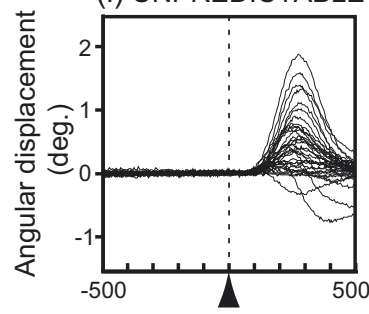

Second Half

(iii) UNPREDICTABLE

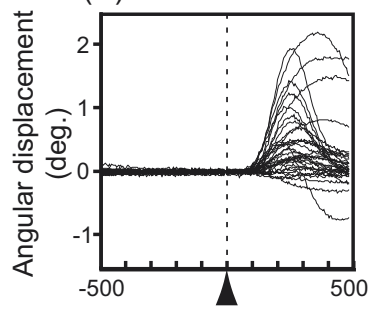

(iv) PREDICTABLE

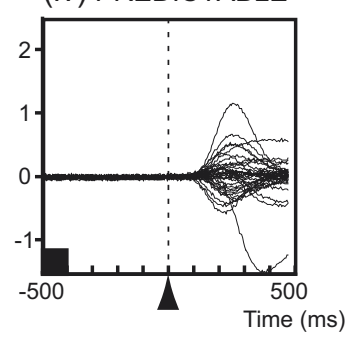

b

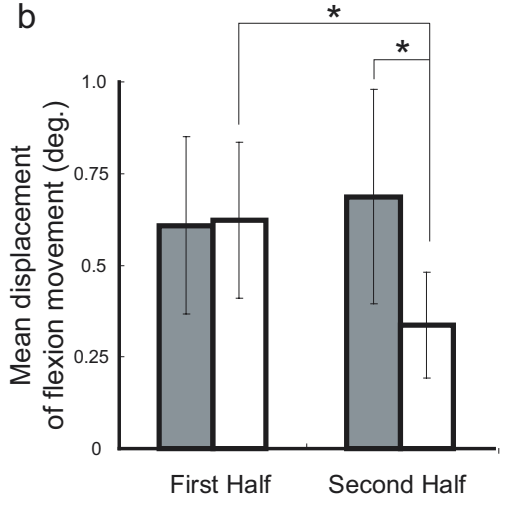

$\square$ UNPREDICTABLE $\square$ PREDICTABLE

Fig. 4. Angular displacements of all movements evoked by TMS (a) and mean displacement of their flexion movements (b). a: Angular displacements of all evoked movements were superimposed. (i) UNPREDICTABLE, first half; (ii) PREDICTABLE, first half; (iii) UNPREDICTABLE, second half; (iv) PREDICTABLE, second half. Vertical axes indicate the directions of movements. Zero (0) in the vertical axes indicates a relaxed hand position. Flexion movements are shown as positive values. Filled squares indicate a cue signal (solid squares), and filled triangles indicate the timing of TMS (solid triangles). b: In the PREDICTABLE condition, the mean displacement of evoked flexion movements became significantly smaller in the second half, but this result was not found in the UNPREDICTABLE condition.

([PREDICTABLE or UNPREDICTABLE] $\times$ [first or second half]) for the MEP amplitude in the FCR muscle showed significant interaction between two factors $(F[1,134]=7.07, p<0.01)$. A test for simple main effect as a post hoc analysis revealed that only in the PREDICTABLE condition were the MEP amplitudes significantly reduced in the second half of the trials when compared with those in the first half $(p<0.05)$. Furthermore, in the second half of the trials the MEP amplitudes in the PREDICTABLE condition were significantly smaller than those in the UNPREDICTABLE condition $(p<0.001)$. No such difference was observed in MEP amplitudes from the ECR (Fig. 3b).

Involuntary movements evoked by TMS. Since the subjects' wrists were not fixed in this experiment, we observed wrist flexion or extension movements just after the TMS. We also found that the directions of movements differ between the first and second halves of the trials, especially in the PREDICTABLE condition (Fig. 4). In the first half of the trials in both the PREDICTABLE and UNPREDICTABLE conditions, most wrist movements were flexion (PREDICTABLE: $81 \%$; mean movement onset $108 \pm 24 \mathrm{~ms}$; UNPREDICTABLE 88\%, $98 \pm 17 \mathrm{~ms}$ ) [Fig. 4a, (i) and (ii)]. Similarly, in the second half of the UNPREDICTABLE condition, most was flexion (75\%) [Fig. 4a, (iii)]. In contrast, flexion or extension in the second half of the PREDICTABLE condition was equally observed (flexion 53\%) [Fig. 4a, (iv)].

The mean displacement of flexion movements became smaller only in the second half of the trials in the PREDICTABLE condition. A two-way ANOVA ([PREDICTABLE or UNPREDICTABLE] $\times$ [first or second half]) for the mean displacement showed significant interaction between the two factors $(F[1,98]=3.8, p=$ $0.055)$. A test for simple main effect revealed that only in the PREDICTABLE condition was the mean displacement of flexion movements significantly reduced in the second half of the trials when compared with the first half $(p<0.05)$. Furthermore, in the second half of the trials the mean displacement in the PREDICTABLE condition was significantly smaller than in the UNPREDICTABLE condition $(p<0.05)$.

\section{DISCUSSION}

The cortico-spinal excitability was reduced when the subjects could predict the timing of TMS that was provided to the hand/wrist section of the motor cortex, even in a situation where the subjects had no intention of making hand movements. We conclude that the predictability of TMS is a crucial factor to reduce the excitability even when no motor set is required.

In the semi-PREDICTABLE condition, the MEP amplitude was gradually reduced as TMS was delivered at a relatively later timing from the $\mathrm{S} 1$ signal (Fig. 2). This gradual reduction of MEP amplitude might, at least partly, relate to the increase of the expectancy of the TMS appearance (i.e., if TMS had not appeared by $375 \mathrm{~ms}$, the probability of its appearance at $500 \mathrm{~ms}$ is 
$100 \%$ ). Thus the reduction of cortico-spinal excitability in the previous studies [6-8], where the subjects had to prepare a response in $\mathrm{S} 1-\mathrm{S} 2$ reaction time paradigm, would partly be explained by the predictability of the TMS timing, since their S1-S2 periods were also relatively short and constant (500 or 1,000 ms) and they could also predict TMS timing. Indeed, when the subjects could not accurately predict the TMS timing during a relatively longer S1-S2 period (from 2 to $6 \mathrm{~s}$ ), the cortico-spinal excitability either slightly increased [4] or was unchanged $[6,8]$, but it was never significantly reduced. Furthermore, Hasbroucq et al. (1999) clearly demonstrated that the reduction of cortico-spinal excitability during an $\mathrm{S} 1-\mathrm{S} 2$ period $(1,000 \mathrm{~ms})$ was not affected by the types of finger movements, and that the reduction was also observed in the relaxed hand that was not used for response. This evidence suggests that not a motor preparation process per se, but a TMS predictability gives rise to the reduction of cortico-spinal excitability in a situation where the subjects can predict the TMS timing.

When TMS timing was completely predictable (PREDICTABLE condition), we also found a significant reduction of MEP amplitude, which was not observed when the timing was totally unpredictable (UNPREDICTABLE condition). More important, this reduction was observed only in the latter part of the trials (Fig. 3). It is very likely that the subjects could precisely predict TMS timings in the second half of the trials even in the same PREDICTABLE condition. Thus the reduction of cortico-spinal excitability in the second half might be due to the precise prediction of TMS timing.

The TMS to the motor cortex at an intensity above the motor threshold quite often evoked involuntary movements, even in a subject that had no intention of making a response, with relatively shorter latencies when compared with voluntary movement responses [14]. In the present study, most involuntary wrist movements evoked by TMS were flexion movements (Fig. 4). At the present wrist flexion angle, the wrist extensor (ECR) muscle was stretched and the flexor (FCR) muscle was relatively shortened. It is known that the excitability of the cortico-spinal pathway increases in the wrist muscle that was passively and statically shortened [10]. Thus the present wrist posture (flexion 60 degrees) that may increase its cortico-spinal excitability provides a better neuronal state in the motor system to evoke involuntary flexion movements by TMS.

In the second half of the trials of PREDICTABLE condition, cortico-spinal excitability was selectively reduced in the flexor muscle, the agonistic muscle for involuntary movements, but not in the extensor (antagonistic) muscle (Fig. 3), to lessen the displacement of involuntary flexion movements (Fig. 4). Thus it seems that the brain reduced the neuronal excitability in the cortico-spinal tract to minimize evoked involuntary movements (when the brain can predict TMS timing).

Involuntary movements or movement perturbations disturb normal neuronal computation in the human motor system. Therefore the muscular-selective reduction of cortico-spinal excitability would be a useful strategy of the brain that may adjust the excitability in a situation where an involuntary movement or a movement perturbation can be predictable in human motor behavior.

This study was supported by the 21st Century COE Program (D-2 to Kyoto University), MEXT, Japan.

\section{REFERENCES}

1. Barker AT, Jalinous $R$, and Freeston IL: Non-invasive magnetic stimulation of human motor cortex. Lancet 1 : 1106-1107, 1985

2. Hallett M: Reaction times. In: Handbook of Transcranial Magnetic Stimulation, ed. Pascual-leone A, Davey NJ, Rothwell J, Wassermann EM and Puri BK, Oxford University Press Inc., New York, pp 339-345, 2002

3. Evarts EV and Tanji J: Reflex and intended responses in motor cortex pyramidal tract neurons of monkey. J Neurophysiol 39: 1069-1080, 1976

4. Hausler UH, Lutzenberger W, and Birbaumer N: Simultaneous recording of slow brain potentials and transcranial magnetic stimulation of hand area in human motor cortex. Neurosci Lett 200: 183-186, 1995

5. Tanji $\mathrm{J}$ and Evarts EV: Anticipatory activity of motor cortex neurons in relation to direction of an intended movement. J Neurophysiol 39: 1062-1068, 1976

6. Hasbroucq T, Kaneko H, Akamatsu M, and Possamai CA: Preparatory inhibition of cortico-spinal excitability: a transcranial magnetic stimulation study in man. Brain Res Cogn Brain Res 5: 185-192, 1997

7. Hasbroucq T, Osman A, Possamai CA, Burle B, Carron S, Depy D, Latour S, and Mouret I: Cortico-spinal inhibition reflects time but not event preparation: neural mechanisms of preparation dissociated by transcranial magnetic stimulation. Acta Psychol (Amst) 101: 243266, 1999

8. Touge T, Taylor JL, and Rothwell JC: Reduced excitability of the cortico-spinal system during the warning period of a reaction time task. Electroencephalogr Clin Neurophysiol 109: 489-495, 1998

9. Oldfield RC: The assessment and analysis of handedness: the Edinburgh inventory. Neuropsychologia 9: 97-113, 1971

10. Lewis GN, Byblow WD, and Carson RG: Phasic modulation of corticomotor excitability during passive movement of the upper limb: effects of movement frequency and muscle specificity. Brain Res 900: 282-294, 2001

11. Naito E: Sensing limb movements in the motor cortexHow humans sense limb movement-. Neuroscientist 9: 73-82, 2004

12. Rossini PM, Barker AT, Berardelli A, Caramia MD, Caruso 
TMS Predictability Reduces Cortico-spinal Excitability

G, Cracco RQ, Dimitrijevic MR, Hallett M, Katayama Y, Lucking $\mathrm{CH}$, et al:: Non-invasive electrical and magnetic stimulation of the brain, spinal cord and roots: basic principles and procedures for routine clinical application. Report of an IFCN committee. Electroencephalogr Clin Neurophysiol 91: 79-92, 1994
13. Brouwer B and Ashby P: Corticospinal projections to upper and lower limb spinal motoneurons in man. Electroencephalogr Clin Neurophysiol 76: 509-519, 1990

14. Brebner JMT and Welford AT: Introduction: an historical background sketch. In: Reaction times, ed. Welford AT, Academic Press, London, New York, pp 1-23, 1980 